\title{
A Kinetic Study of the Mode of Growth of Surface Colonies of Bacteria and Fungi
}

\author{
By S. J. PIRT \\ Department of Microbiology, Queen Elizabeth College, University of \\ London, Campden Hill, London, W.8
}

(Accepted for publication 2 December 1966)

\begin{abstract}
SUMMARY
A model for the growth of microbial colonies on the surface of a solid nutrient medium is discussed. The model accounts for the constant rate of increase in the colony radius which is characteristic of a fungal colony growing on the surface of a nutrient medium.

Experiments showed that bacterial colonies after about $12 \mathrm{hr}$ of development showed a virtually constant rate of radial growth over a $12 \mathrm{hr}$ period. Over longer periods ( $24 \mathrm{hr}$ ) a gradual decline in the colony radial growth rate was apparent. The initial rate of radial growth of the bacterial colony was a useful parameter of the growth rate of the organism. The effects on the initial colony radial growth rate of the following factors were determined: initial nutrient concentration depth of agar layer; maximum specific growth rate (ln $2 /$ minimum doubling time); oxygen partial pressure; humidity of gas phase; temperature. Three bacterial types, Escherichia coli, Klebsiella aerogenes and Streptococcus faecalis were studied. With $E$. coli growing in minimal medium in air at $1 \mathrm{~atm}$. pressure when the growth was glucoselimited, oxygen became a limiting factor when the glucose concentration exceeded $0.25 \%(\mathrm{w} / \mathrm{v})$. With a glucose concentration of $1 \%(\mathrm{w} / \mathrm{v})$, the growth was strongly inhibited, probably by toxic products.

When the colony growth was glucose-limited and oxygen was present in excess, the relation between initial colony radial growth rate $\left(K_{r}\right)$, the initial glucose concentration $\left(s_{0}\right)$ and the maximum specific growth rate $\left(\alpha_{m}\right)$ was

$$
K_{r}=k_{2}\left(\sqrt{ } s_{0}-\sqrt{ } s_{i}\right) \sqrt{ } \alpha_{m},
$$

where $k_{2}$ is a constant; $s_{i}$, called the 'lag concentration', is a value of the glucose concentration which must be exceeded before growth of the colony can occur. The value of $s_{i}$ was very small or negligible except with a certain type of inhibitory condition, such as an over-optimal concentration of oxygen, which could be overcome by the organism's metabolic activity. Direct proportionality between $K_{r}$ and $\sqrt{ } \alpha_{m}$ was found by varying the maximum specific growth rate by adding sulphanilamide. When $\alpha_{m}$ was varied by temperature changes the linear relation between $K_{r}$ and $\sqrt{ } \alpha_{m}$ did not hold. The implications of these results and their potential applications are discussed.
\end{abstract}

\section{INTRODUCTION}

Although the growth of fungi or bacteria in colonies on the surface of a solid nutrient medium is a general experimental technique and of great ecological importance, the laws which govern colonial growth have not been elucidated. The fact that we expect a colony of a given organism to reach a certain size and shape in a certain 
time implies that there are underlying laws. We need to know what factors govern the rate of growth of the colony, how the outward spread of the colony is related to mass growth, what governs the ultimate size of the colony, whether all parts of the colony grow at the same rate and if not, what causes the differential growth. The solution of these problems should extend the usefulness of the colony-growth technique and facilitate quantitative studies of microbial ecology. The object of the present paper is to provide a theoretical and experimental analysis of some of these problems.

The basis on which the concepts of colony growth are built are the growth laws derived from study of the growth of populations of organisms in submerged homogeneous liquid cultures. In such cultures, as long as all nutrients are present in excess and growth inhibitors are not accumulated, the organisms grow exponentially. The law for such growth is:

$$
\frac{d M}{d t}=\alpha M
$$

where $M=$ organism mass/unit volume, $t=$ time, and $\alpha$ is a constant known as the 'specific growth rate'. Integration of (1) gives the well-known logarithmic relation for growth

$$
\ln M=\alpha t+\ln M_{0},
$$

where $\ln =$ natural logarithm and $M_{0}=$ concentration of organisms at zero time. The specific growth rate $(\alpha)$ is a most useful parameter because it is a direct measure of the growth rate of a population in a given environment. It is related to the doubling time $\left(t_{d}\right)$ of the organisms by the expression $t_{d}=\ln 2 / \alpha$. It should be pointed out that exponential growth is characteristic not only of bacteria, yeasts and other nonfilamentous organisms, but also of filamentous fungi provided that these filaments are homogeneously dispersed and not aggregated into 'pellets' (Pirt, 1966).

If the flow of nutrients and oxygen into a microbial colony were unrestricted and growth inhibitors did not accumulate, the growth of the colony should conform to (1). Suppose the colony grew as a hemisphere of radius $r$ with a constant mass of organisms $\rho / \mathrm{cm}^{3}$. Substituting $M=\frac{2}{3} \pi r^{3} \rho$ in (1), then

$$
\ln r=\frac{\alpha}{3} \mathrm{t}+\ln r_{0},
$$

where $r_{0}$ is the radius of the colony at zero time. If the colony grew as a disc of constant height $h$ and radius $r$, then $M=\pi r^{2} h \rho$. Substitution of this value in (1) leads to

$$
\ln r=\frac{\alpha}{2} t+\ln r_{0}
$$

In either case, therefore, the radius should increase exponentially and the slope of the plot of $\ln r$ against $t$ should be linearly related to the specific growth rate. But it is common experience that microbial colonies of visible size growing on the surface of a solid nutrient do not spread outwards at an exponential rate.

Virtually all the work on colony growth rates has been concerned with filamentous fungi; there seem to be no reports of quantitative studies on the kinetics of growth of bacterial or yeast colonies. It is commonly accepted that colonies of filamentous fungi growing on solid media in Petri-dish cultures spread outwards at a constant rate, often termed the 'linear rate of growth'. Fawcett (1925) apparently was the first who explicitly observed that fungal colonies spread at a constant rate along the sur- 
face of a nutrient agar contained in a tube. The linear rate of colony spread along a tube was used by Ryan, Beadle \& Tatum (1943) as a parameter of the growth rate of Neurospora. Other workers (e.g. Brancato \& Golding, 1953) have used the constant rate of increase in the radius of a fungal colony on a Petri-dish culture as a parameter of the growth rate. A detailed morphological and quantitative study of colonies of a fungus Chaetomium sp. was reported by Plomley (1959), who found that initially the radius of the colony increased exponentially and the transition to a constant radial growth rate occurred at a diameter of about $0.2 \mathrm{~mm}$.

The constant rate of increase in colony radius is represented as follows. If $r$ is the colony radius at time $t$, and $r_{0}$ at time zero, then

$$
r=K_{r} t+r_{0}
$$

where $K_{r}$ is a constant. Since $K_{r}$ represents the increase in the radius per unit time, $K_{r}$ is here called the 'radial growth rate'.

An estimate of the height of the colony can be derived from the analysis by Hill (1928) of nutrient diffusion into a layer of cells such as a tissue. From this analysis it follows that the thickness $\left(h_{g} \mathrm{~cm}\right.$.) to which a diffusing nutrient will penetrate a metabolizing tissue is limited to

$$
h_{g}=\sqrt{\frac{2 D s_{0}}{q}}
$$

where $D\left(\mathrm{~cm} .{ }^{2} / \mathrm{sec}\right.$.) is the diffusion constant for the nutrient, $s_{0}\left(\mathrm{~g} . / \mathrm{cm} .^{3}\right)$ is the concentration of nutrient at the surface and $q$ is the metabolic quotient (g. nutrient consumed $/ \mathrm{cm}^{3}$ of tissue $/ \mathrm{sec}$.). The maximum value of $q$ is given by $q=\alpha_{m} / Y$ (Pirt, 1957), where $\alpha_{m}\left(\mathrm{sec}^{-1}\right)$ is the maximum specific growth rate of the organisms and $Y$ (cm. ${ }^{3}$ tissue formed $/ \mathrm{g}$. nutrient) is the yield. The minimum value of $q$ will be the maintenance requirement (Pirt, 1965). The minimum and maximum values for the thickness $\left(h_{g}\right)$ of the growing layer in an Escherichia coli surface colony may be estimated as follows. A colony growing on a glucose minimal medium agar with growth limited by the glucose supply is considered. The glucose concentration is taken as $2.5 \mathrm{~g} .11$., that is, $s_{0}=2.5 \times 10^{-3} \mathrm{~g}$. glucose $/ \mathrm{cm}^{3}, D=0.6 \times 10^{-5} \mathrm{~cm} .{ }^{2} / \mathrm{sec}$. $Y=4.4 \mathrm{~cm}^{3} / \mathrm{g}$. glucose (calculated from a dry weight yield of $0.44 \mathrm{~g} . / \mathrm{g}$. glucose, and a population density (concentration of organism) equivalent to $0.1 \mathrm{~g}$. dry wt. organism $/ \mathrm{cm}^{3}$ ), $\alpha_{m}=1.94 \times 10^{-4} \mathrm{sec}^{-1}$ (that is, $0.70 \mathrm{hr}^{-1}$ ). Hence the minimum value calculated for the thickness of the growing layer of the colony is $260 \mu$. The maintenance energy requirement may be taken as about one-tenth of the glucose requirement at the maximum growth rate, therefore the maximum value of $h_{g}$ will be about three times the minimum value, that is, $780 \mu$. In fact, the concentration of glucose at the colony surface will be lower than the initial concentration, hence the above values for $h_{g}$ must be overestimates.

An estimate of the maximum thickness of the growing layer of a colony of, say, Escherichia coli may also be determined from the oxygen requirement. In relation (6) above the following values are inserted: $D=1.9 \times 10^{-5} \mathrm{~cm} .{ }^{2} / \mathrm{sec}$.; $s_{0}=8 \times 10^{-6}$ g. $/ \mathrm{cm}^{3}$ (for water saturated with oxygen at $0.21 \mathrm{~atm}$. pressure); $q=2 \times 10^{-5} \mathrm{~g}$. oxygen $/ \mathrm{cm}^{3}$ colony $/ \mathrm{sec}$. (calculated on the basis that the $q_{\mathrm{o}_{2}}=500 \mathrm{ml}$. oxygen $/ \mathrm{g}$. organism dry wt./ hr and that there is $0.1 \mathrm{~g}$. organism dry wt. $/ \mathrm{cm}^{3}$ colony). The value for $h_{g}$ then is $40 \mu$. Even if the cells were only respiring at the maintenance level (Pirt, 1965) the $q_{\mathrm{o}_{2}}$ would be about $2 \times 10^{-6} \mathrm{~g}$. oxygen $/ \mathrm{cm} .^{3} / \mathrm{sec}$. and the maximum 
value of the depth to which oxygen would penetrate would be about $127 \mu$. It is therefore concluded that, since the thickness of the growing layer of bacteria will be small and constant in the steady state, the total height of the colony will not vary greatly.

To account for the growth rate of a microbial colony the following working hypothesis was developed. Growth of a colony on the surface of a solid homogeneous medium such as a nutrient agar was considered, and growth into the medium was excluded. It was supposed that if the inoculum consisted of one or a few organisms, then they would receive nutrients at concentrations above the growth-limiting values and consequently, initially the colony would grow at an exponential rate. In the

(a)

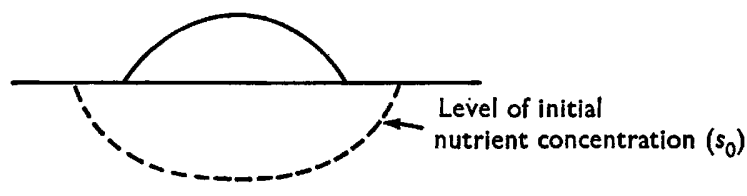

(b)

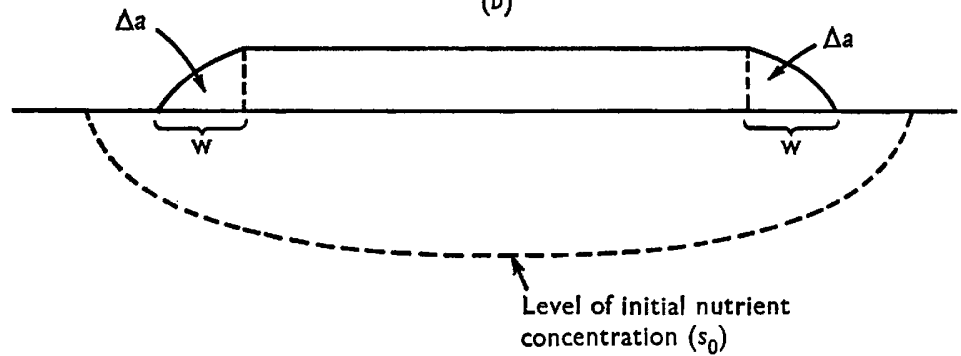

Fig. 1. Vertical cross-sections through model colonies (not to scale): (a) during initial exponential growth; $(b)$ during phase of constant radial growth rate. The area $\Delta a$ of width $w$ represents the postulated zone of growth.

exponential phase it is concluded that growth will occur equally in all directions on the surface and lead to a hemispherical shape for the colony (see Fig. 1a). A gradient of nutrient concentration will develop under the colony. This means that the level in the agar at which the nutrient concentration has the initial value $s_{0}$, will recede from the agar surface as shown in Fig. $1 a$. With increase in size of the colony it was supposed that increase in nutrient demand and decrease in nutrient diffusion rate would occur as the colony grew thicker, and that eventually the concentration of some nutrient, termed the 'growth-limiting nutrient', would decrease virtually to zero in the centre of the colony and stop growth there. Then growth would be restricted to an outer peripheral zone of the colony. This state of the colony is represented in Fig. $1 b$. In the model it was assumed that ultimately a near steady state would be set up in which the width $(w)$ of the growing zone remained constant as shown in Fig. $1 b$. The height of the colony was assumed to have the constant value $(h)$ except in the peripheral growing zone. The colony growth rate according to this model is derived as follows. Let $M=$ total mass of the organisms in the colony; $M_{g}=$ mass of organisms in the 
growing zone with constant specific growth rate, $\alpha$; then the mass growth rate of the colony is given by

$$
\frac{d M}{d t}=\alpha M_{g} .
$$

When the width $w$ of the growing zone is small compared with the colony radius $r$, to a close approximation, $M=\rho \pi r^{2} h$, where $\rho=$ organism mass $(\mathrm{g}.) / \mathrm{cm} .^{3}$ colony. Also $M_{\theta}$ approximates to $\rho 2 \pi r \Delta a$, where $\Delta a$ is the cross-sectional area of the growing zone and is taken to be constant. Substituting these values in (7) leads to

Hence

$$
\frac{d r}{d t}=\frac{\Delta a}{h} \alpha .
$$

$$
r=\frac{\Delta a}{h} \alpha t+r_{0},
$$

where $\Delta a, h$ and $\alpha$ are taken to be constants. Thus according to this model the colony should have a constant radial growth rate equal to $(\Delta a) / h$. If $\Delta a$ approximates to a triangle of area $w h / 2$, then (9) becomes

$$
r=\frac{w \alpha}{2} t+r_{0} .
$$

Development of the model requires a quantitative analysis of the problem of nutrient diffusion into the colony. However, despite this lack, the present theory has served as a useful guide to experiments designed to elucidate factors which control the growth rate of bacterial colonies.

\section{METHODS}

Medium. Defined medium A (DMA) contained the following constituents (amounts/ 1.): $\mathrm{K}_{2} \mathrm{HPO}_{4}, 11 \cdot 3 \mathrm{~g}$.; $\mathrm{NaH}_{2} \mathrm{PO}_{4}, 2 \mathrm{H}_{2} \mathrm{O}, 5 \cdot 4 \mathrm{~g}$.; $\mathrm{MgSO}_{4} .7 \mathrm{H}_{2} \mathrm{O}, 200 \mathrm{mg}$.; $\mathrm{CaCl}_{2}, 10 \mathrm{mg}$.; $\mathrm{FeSO}_{4} .7 \mathrm{H}_{2} \mathrm{O}, 5 \mathrm{mg}$.; $\mathrm{ZnSO}_{4} .7 \mathrm{H}_{2} \mathrm{O}, 0.5 \mathrm{mg}$.; $\mathrm{MnSO}_{4} \cdot 4 \mathrm{H}_{2} \mathrm{O}, 0.5 \mathrm{mg}$.; $\mathrm{CuSO}_{4} .5 \mathrm{H}_{2} \mathrm{O}$, $0.1 \mathrm{mg}$.; $\mathrm{CoCl}_{2} .6 \mathrm{H}_{2} \mathrm{O}, 0.1 \mathrm{mg}$.; sodium borate, $0 \cdot 1 \mathrm{mg}$.; sodium molybdate, $0.1 \mathrm{mg}$.; ethylenediaminetetra-acetic acid (EDTA), $0.26 \mathrm{~g}$.; $\mathrm{NH}_{4} \mathrm{Cl}, 2.0 \mathrm{~g}$. 1 .; glucose, amounts separately specified. The final $\mathrm{pH}$ value was $7 \cdot 4$.

Medium DMA was prepared in the following manner. The phosphates were mixed at $\times 10$ final strength and adjusted with $\mathrm{NaOH}$ to $\mathrm{pH} 7 \cdot 50-7 \cdot 55$. The buffer with the other constituents gave finally $\mathrm{pH} 7 \cdot 4$.

The $\mathrm{Mg}$ and $\mathrm{Ca}$ salts were dissolved in one solution with an equivalent amount of EDTA and adjusted to $\mathrm{pH} 7-8$ with $\mathrm{NaOH}$; the stock solution was $100 \times$ final concentration. The iron, zinc, manganese, copper, cobalt, borate and molybdate salts were dissolved one by one in an equimolar amount of EDTA adjusted to $\mathrm{pH}$ 7-8 with $\mathrm{NaOH}$, and the stock solution was adjusted to $100 \times$ final concentration. There was evidence that when the stock solutions were kept more than 6 months the growth rate of Escherichia coli in the medium declined.

The phosphate and the mineral salts were combined for autoclaving at $121^{\circ}$ for $15 \mathrm{~min} . \mathrm{NH}_{4} \mathrm{Cl}$ and glucose were each sterilized separately by autoclaving at $121^{\circ}$ for $15 \mathrm{~min}$.

The PYGS medium consisted of the same constituents as medium DMA except that $\mathrm{NH}_{4} \mathrm{Cl}$ was replaced by peptone $(5 \mathrm{~g} . / \mathrm{l}$.) + Difco yeast extract $(3 \mathrm{~g} . / 1$.$) .$

Preparation of agar plates. To prepare the solid medium for plate cultures, $10 \mathrm{ml}$. 
of double strength DMA + glucose medium at about $50^{\circ}$ and $10 \mathrm{ml}$. of molten $2 \%$ (w/v) agar (New Zealand) were mixed in a tube and poured into a standard glass Petri dish of $9 \mathrm{~cm}$. diameter. The pipette used for the agar was calibrated to allow for the large drainage error (about $0.5 \mathrm{ml}$.). Before filling the Petri dishes they were levelled on a tripod table with levelling screws. The levelling table consisted of a piece of plate glass held at each side by a slotted piece of wood carrying the levelling screws. After the agar had set, the plates were left with the lids on for $2-4 \mathrm{hr}$ in a $37^{\circ}$ constant-temperature room. The plates were then dried in the inverted position, with the lids removed. The drying time in our hot room which had a relative humidity of about $30 \%$ was 3-6 min. A useful indication of the completion of drying was the disappearance of the droplets of condensate on the Petri-dish lid. After drying the plates were used immediately.

Inoculation of plates. A satisfactory method of inoculating plates was gradually evolved during the course of the experiments. Initially organisms were spread on the plates as for a plate count but this was abandoned because on the DMA + glucose agar medium there was a lag of one or more days before the colonies were visible. Growth from a mass inoculum was preferred because it eliminated lag due to a small inoculum, and it enabled the colonies to be spaced evenly on the agar. Attempts to produce streaks or pinpoint inocula with inoculating needles did not produce sufficiently even streaks or round colonies. The method finally used was to pipette a small drop of a dense culture on to the agar surface. Fine uniform-bore capillaries which delivered about $2 \mu \mathrm{l}$. by means of a teat were at first used. With this type of pipette the variation in volume delivered might be several-fold, but its chief disadvantage was the impossibility of avoiding spray formation which caused many satellite colonies to develop near the main one and thus interfere with its development. A good tool for the inoculation was the Repette (Jensons, Hemel Hempstead, Herts., England) which dispensed $\mu \mathrm{l}$. amounts by means of a plunger action. The Repette was dismantled for sterilization by autoclaving or by immersion in boiling water and then assembled aseptically. The Repette, adjusted to deliver $3 \mu 1$. drops was discharged on to the agar by gently bringing the drop in contact with the agar. Four drops, one at each corner of a square about $2.5 \mathrm{~cm}$. apart, were dispensed on each plate; in some experiments a fifth drop was placed at the middle of the square. The method was rapid and the drop size highly reproducible. After inoculation the plates were placed in the incubator, lids down.

Measurement of colony size. The diameters of colonies were at first measured by means of a low-power miroscope with a micrometer eyepiece. However, this method proved tedious, especially since it had to be done in the $37^{\circ}$ room; it also involved removing the Petri-dish lids, with consequent drying of colonies and risk of infection. A most convenient method of measuring the colony size accurately was to use an enlarger with a projection screen (the Shadomaster, supplied by Buck and Hickman, Otterspool Way, Watford, Herts., England). By this means colony size was magnified ten times and the size measured on the screen with a transparent ruler. There was no need to remove the lid of the Petri dish, and since the sizes of the colonies on a plate could be measured within $30 \mathrm{sec}$. each plate could be removed from the incubator for the duration of the measurement. The mean of two colony diameters at right angles was taken for each colony.

Shake-flask cultures. Homogeneous liquid aerobic cultures were obtained by growth 
in $250 \mathrm{ml}$. conical flasks on a rotary shaker. The flasks initially contained $25 \mathrm{ml}$. medium $+1 \mathrm{ml}$. inoculum. Growth was followed by measurement of the culture opacity.

Inocula. The organisms used were Escherichia coli (laboratory strain в 3), Klebsiella aerogenes NCIB 8017 (National Collection of Industrial Bacteria, Torrey Research Station, Aberdeen, Scotland) and Streptococcus faecalis (laboratory strain, D 13).

To obtain reproducible inocula the following procedure was used. First a tube of nutrient broth $(5 \mathrm{ml}$.) was inoculated with a loopful of organisms from a slope culture. When the broth culture was obviously turbid, usually at 3-4 hr, the nutrient broth culture $(5 \mathrm{ml}$.) was transferred to $10 \mathrm{ml}$. of the final medium + glucose $(0.5 \%, \mathrm{w} / \mathrm{v})$ in a $250 \mathrm{ml}$. conical flask. This was incubated without shaking for 4-8 hr. From this second stage $0.5 \mathrm{ml}$. was transferred to $20 \mathrm{ml}$. final medium + glucose $0.5 \%(\mathrm{w} / \mathrm{v})$, in a $250 \mathrm{ml}$. conical flask, and incubated overnight on a rotary shaker at about 200 rev. $/ \mathrm{min}$. with a $1.5 \mathrm{in} .(3.8 \mathrm{~cm}$.) throw. This third-stage inoculum, which contained about $4 \times 10^{9}$ organisms $/ \mathrm{ml}$., was used to inoculate either shake-flask cultures or plate cultures.

\section{RESULTS}

\section{Radial growth rate of bacterial colonies}

DMA nutrient agar plates were inoculated with Escherichia coli at four or five evenly spaced points. The plates were incubated for $9-12 \mathrm{hr}$ to produce confluent growth in each colony; the colony diameters were then measured at intervals of 3 or $4 \mathrm{hr}$. The rate of increase in the diameters of replicate colonies at two different glucose concentrations are shown in Fig. 2. The data for E. coli show that over the initial period of measurement, from 12 to $24 \mathrm{hr}$ after inoculation, the diameters increased at a virtually constant rate which was independent of the colony size over the range studied (2-8 mm. diameter). The term 'initial radial growth rate' is used to refer to the rate of increase in the radius $(\mu / \mathrm{hr})$ in roughly the first $12 \mathrm{hr}$ after the growth in the colony became confluent. The standard deviation in the initial radial growth rates was $7 \%$ of the mean with $0.5 \%(\mathrm{w} / \mathrm{v})$ glucose and $8 \%$ with $0.1 \%(\mathrm{w} / \mathrm{v})$ glucose (the radial growth rate was a function of the glucose concentration). In each experiment the mean radial growth rate of 8-12 replicate colonies was determined; thus the standard deviation in the mean was about $3 \%$. The variation in the mean radial growth rate from different experiments was about $6 \%$. This increase in the variance is attributed to batch-to-batch variations in media and inocula. The specific growth rate in shake-flask cultures varied to a similar degree with different batches of medium and inoculum.

Over longer periods of time ( $36 \mathrm{hr}$ after inoculation) a decrease in the radial growth rate was apparent. With Escherichia coli the radial growth rate in the period 24-36 $\mathrm{hr}$ after inoculation was about $20 \%$ less than in the period $12-24 \mathrm{hr}$, but with increasing time of incubation the rate of decrease in the radial growth rate decreased. Similar results were obtained with colonies of Klebsiella aerogenes and Streptococcus faecalis.

\section{Effect of agar depth and concentration}

It was found that decreasing the agar concentration from 1.0 to $0.6 \%$ had no effect on the colony growth rate.

To determine the effect of the depth of the agar layer, plates were poured with differ- 
ent volumes of nutrient agar $(5,10,15,20,25 \mathrm{ml}$., corresponding to depths of $0 \cdot 86,1 \cdot 72$, $2 \cdot 58,3.44,4.30 \mathrm{~mm}$., respectively). Escherichia coli colonies were grown on the plates and the radial growth rates determined. At each agar depth constant radial growth rates were obtained over a $12 \mathrm{hr}$ period. The results (Fig. 3) showed that the agar

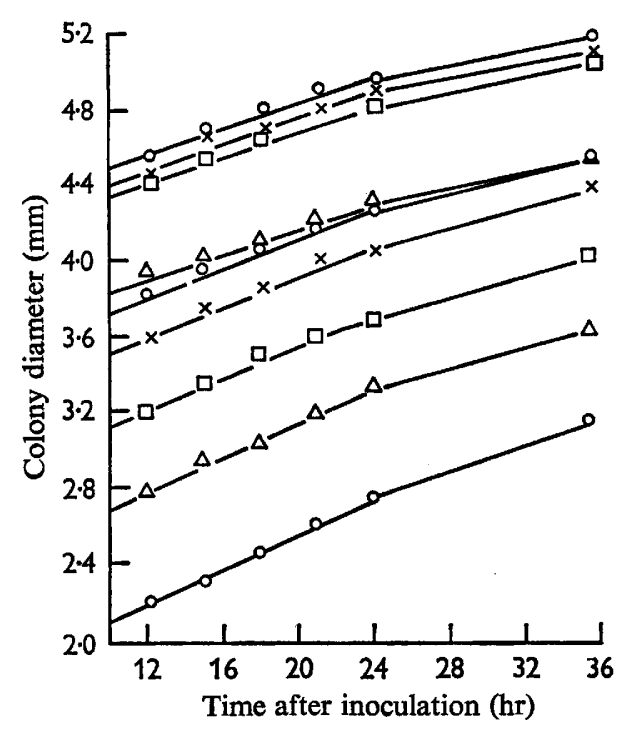

$a$

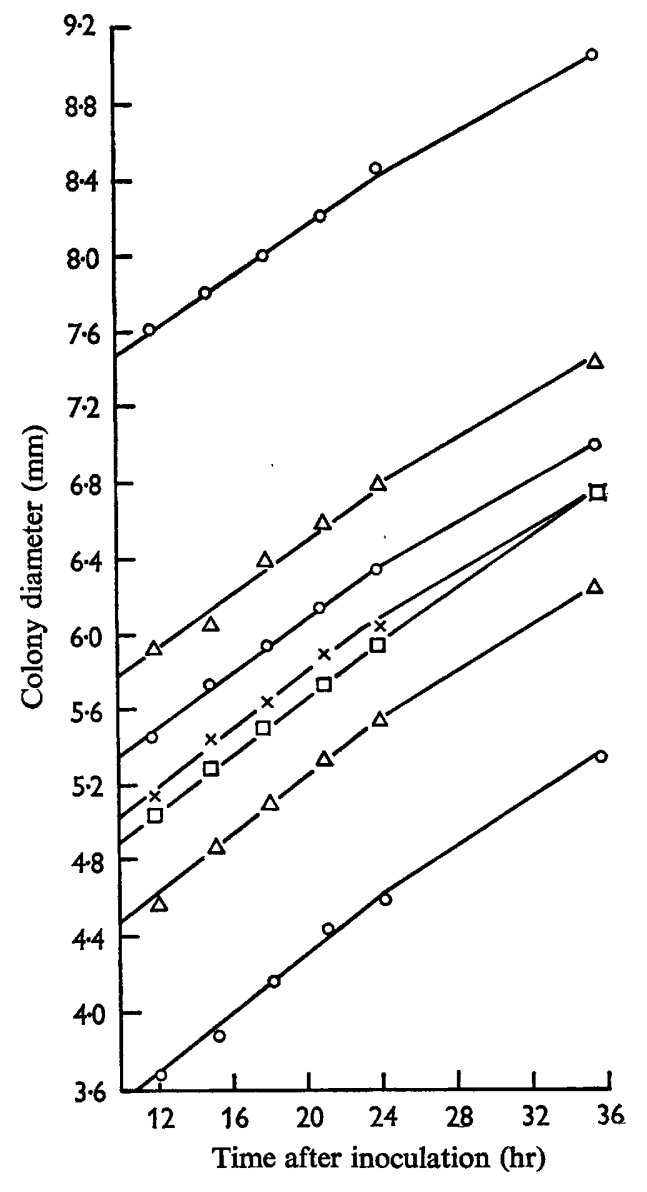

$b$

Fig. 2. Rates of increase in the diameters of Escherichia coli colonies on nutrient agar: (a) glucose, $1.28 \mathrm{~g} . / 1$; (b) glucose, $5.12 \mathrm{~g} . / 1$. Medium DMA; temperature $37^{\circ}$.

depth up to $3.44 \mathrm{~mm}$. (20 ml. agar) had a marked effect on the radial growth rate of colonies. With $0.1 \%(\mathrm{w} / \mathrm{v})$ glucose there was little further increase in growth rate on increasing the agar depth above $3.5 \mathrm{~mm}$. This result indicated that the glucose concentration gradient in the agar extended to a depth of about $3.5 \mathrm{~mm}$.

\section{Effect of humidity}

The good reproducibility of the colony radial growth rates in different plates and different experiments indicated that the degree of drying of the plates was either invariable or immaterial. The effect of the relative humidity of the gas-phase on the colony growth rates over a period of 2-3 days was investigated by growing replicate 
plates of Escherichia coli colonies both in the hot room with a relative humidity of $30 \%$ and in a sealed chamber with a relative humidity of $100 \%$. A layer of water was kept in the chamber (normally a desiccator) to saturate the atmosphere. The watersaturated chamber was kept in the hot room at $37^{\circ}$. The growth rates of $E$. coli colonies over periods of 2 days were virtually the same at both relative humidities. Hence it is concluded that any loss of water from the plates which occurred during drying was immaterial.

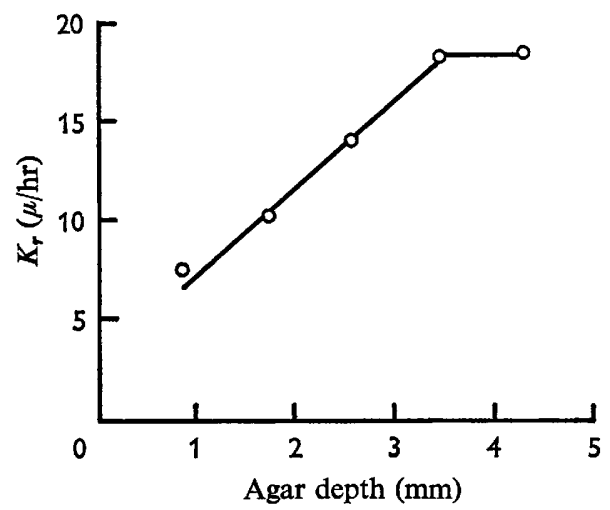

Fig. 3. Effect of agar depth on initial radial growth rates $\left(K_{r}\right)$ of Escherichia coli colonies. Temperature $37^{\circ}$; medium DMA + glucose $(1 \cdot 0$ g. $/ 1$.$) .$

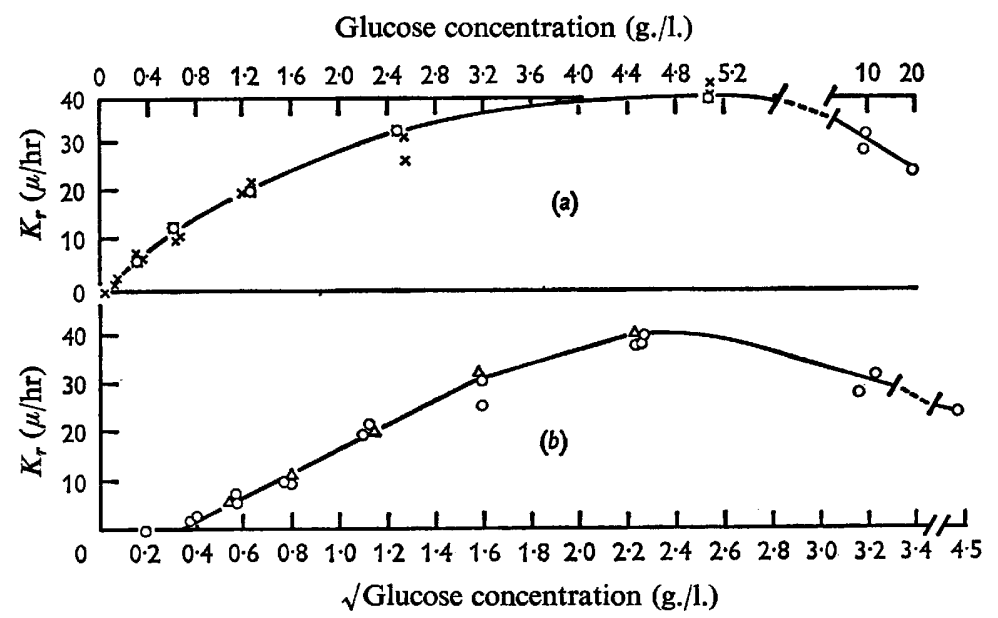

Fig. 4. Initial radial growth rates $\left(K_{\tau}\right)$ of Escherichia coli colonies as functions of the initial glucose concentration. (a) $K_{r}$ plotted against the glucose concn.; $\times$, in air; $O$, in air $+4.5 \%$ ( $v / v) \mathrm{CO}_{2}$. (b) $K_{r}$ plotted against the square root of the glucose concn.; $O$, in air; $\triangle$, in air $+4.5 \%(\mathrm{v} / \mathrm{v}) \mathrm{CO}_{2}$. Medium DMA; temperature $37^{\circ}$.

\section{Influence of glucose concentration}

Plate cultures were set up with different concentrations of glucose, in order to determine the range of glucose concentrations over which the initial radial growth rate was glucose-limited.

The effects of glucose concentration on the initial radial growth rates $\left(K_{r}\right)$ of 
Escherichia coli, Klebsiella aerogenes and Streptococcus faecalis in air are shown in Figs. 4,5 and 6. The graphs of $K_{r}$ against glucose concentration show two main features: (1) at the low glucose concentration the radial growth rate was glucose-limited; (2) at glucose concentrations of $10 \mathrm{~g} . / 1$. or more the colony radial growth was strongly inhibited in the cases of $E$. coli and S. faecalis. Colony radial growth of $K$. aerogenes was not inhibited by glucose at $10 \mathrm{~g}$./I.; probably it would be at still higher glucose concentrations.

\section{Glucose concentration (g./1.)}

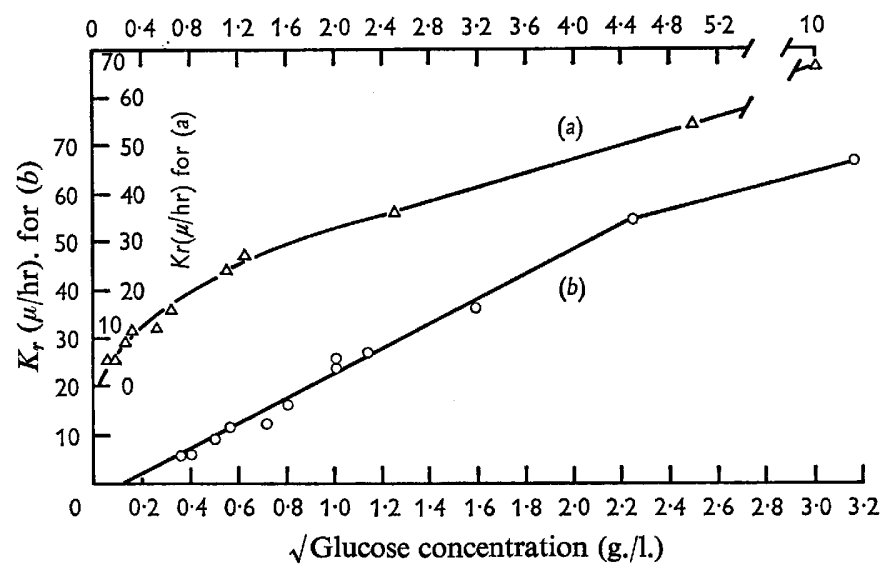

Fig. 5. Initial radial growth rates $\left(K_{r}\right)$ of Klebsiella aerogenes colonies as functions of the initial glucose concentration. $(a) K_{r}$ plotted against glucose concentration, $\triangle ;(b) K_{r}$ against the square root of the glucose concn. $O$, Medium DMA; temperature $30^{\circ}$.

\section{Glucose concentration (g./1.)}

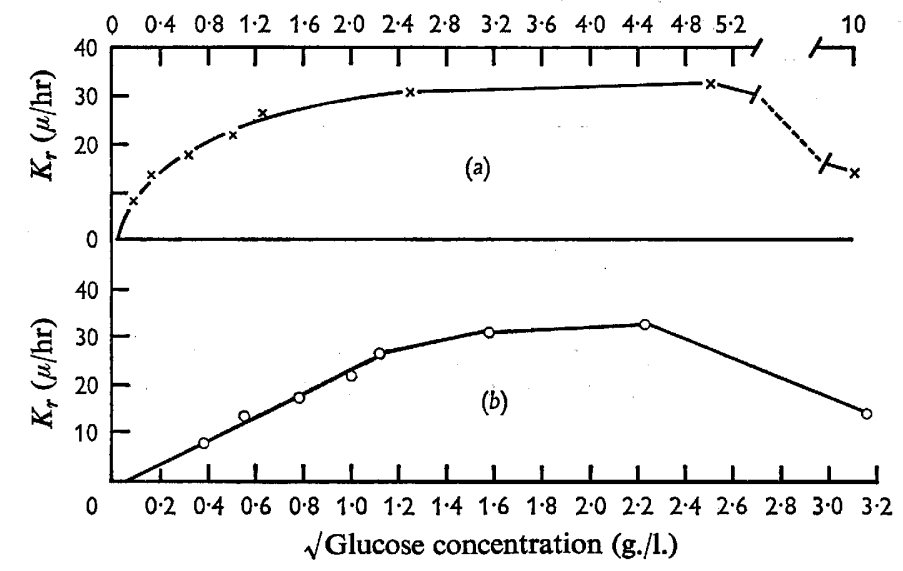

Fig. 6. Initial radial growth rates $\left(K_{r}\right)$ of Streptococcus faecalis colonies as functions of the initial glucose concentration. (a) $K_{r}$ plotted against the glucose concentration $(\times)$; $(b) K_{r}$ against the square root of the glucose concentration $(O)$. Medium PYGS; temperature $37^{\circ}$.

The relation between the initial glucose concentration and the initial colony radial growth rate is not of the Michaelis-Menten type which one finds in homogeneous liquid cultures (Monod, 1942). This is to be expected because in the developed colony the 
initial glucose concentration cannot be the concentration to which the bacteria are exposed, because of the concentration gradient which must develop in the agar. Also it is apparent that, whereas in liquid culture Escherichia coli reaches half its maximum growth rate at about $4 \times 10^{-3} \mathrm{~g}$./1. (Monod, 1942) the value of the initial glucose concentration at which the radial growth rate of the $E$. coli colony reached half its maximum value was $1.3 \mathrm{~g}$. $/ 1$. The relation between the initial colony growth rate and the square root of the initial glucose concentration for each of the three organisms (Figs. 4, 5, 6) was linear over a considerable range. This range for $E$. coli was $0-2 \cdot 5$ g./1., for $K$. aerogenes $0-5 \mathrm{~g}$./1. and for $S$. faecalis $0-1 \cdot 25 \mathrm{~g}$./1. In the case of $E$. coli it is shown below that at $2.5 \mathrm{~g}$. glucose/l. oxygen became the growth-limiting factor. For similar reasons it is believed that oxygen became the growth-limiting factor for $K$. aerogenes when the glucose concentration reached $5 \mathrm{~g} . / 1$. In the case of $S$. faecalis the departure from the linear relation between the initial radial growth rate and the square root of the initial glucose concentration may have been due to some nutrient other than glucose, possibly an amino acid or a vitamin, becoming growthlimiting. If this were so, then increasing the concentration of peptone or yeast extract should increase the maximum glucose concentration at which growth is limited.

A surprising feature was the strong inhibition of growth of Escherichia coli and of Streptoccocus faecalis caused by glucose at $10 \mathrm{~g} . / 1$. This inhibition is attributed to the formation of toxic products or change in the $\mathrm{pH}$ value of the medium. We can compare the colony growth rates of colonies of $E$. coli and $S$. faecalis under glucose limitation when the initial glucose concentration was $1.0 \mathrm{~g} . / 1$. At the optimum temperature, $37^{\circ}$, the $K_{r}$ for $E$. coli was $20 \mu / \mathrm{hr}$, and for $S$. faecalis $23 \mu / \mathrm{hr}$. One of the classical distinguishing features of the lactic acid bacteria of which $S$. faecalis is one, is that they normally produce minute colonies as a result of low colony growth rates. It seems probable that this may be due, not so much to an intrinsically lower colony growth rate, but to the strong inhibition caused by the practice of using a high glucose concentration ( 1 or $2 \%, w / v)$ in the normal plate culture media for lactic acid bacteria.

Figures 4, 5 and 6 show that the initial radial growth rates of colonies reached zero at a certain small, but finite, glucose concentration termed the 'lag concentration', $s_{i}$. For Escherichia coli in air, $s_{i}$ was $0.090 \mathrm{~g} . / 1$., for Klebsiella aerogenes, $0.013 \mathrm{~g} . / 1$. and for Streptococcus faecalis, 0.005 g./1. A possible cause of this minimum value for the glucose concentration before growth could occur is discussed below. The results show that the relation between the glucose concentration and the initial radial growth rate $\left(K_{r}\right)$ was of the form

$$
K_{r}=k_{1}\left(\sqrt{ } s_{0}-\sqrt{ } s_{i}\right),
$$

where $k_{1}$ is a constant. The value of $s_{i}$ in some cases would be small enough to be negligible.

\section{Oxygen and carbon dioxide limitation of colony growth rate}

To see whether oxygen was limiting the growth of Escherichia coli colonies, growth in air and in oxygen at one atmosphere pressure was compared. To obtain the different gas-phases the plates were put in a sealed jar of the type normally used for anaerobic culture (vacuum desiccator vessels also suggest themselves for this purpose) and the gas in the vessel was changed by evacuation and filling with the appropriate gas mixture. A spacer (Khairat, 1963) was included between the lid and the base of each Petri 
dish to ensure that the gas circulated. Colonies were first allowed to develop in air, then after measurement they were transferred to the appropriate gas phase.

- The effect of oxygen concentration on the radial growth rate of Escherichia coli colonies is shown in Fig. 7. The surprising feature is that at low glucose concentrations $(0-1.7 \mathrm{~g} . / 1$.$) oxygen at 1 \mathrm{~atm}$. pressure almost completely inhibited the growth of the colonies. With glucose above the inhibitory concentration the radial growth rate responded to glucose concentration in oxygen (1 atm. pressure) in the same way as in air;

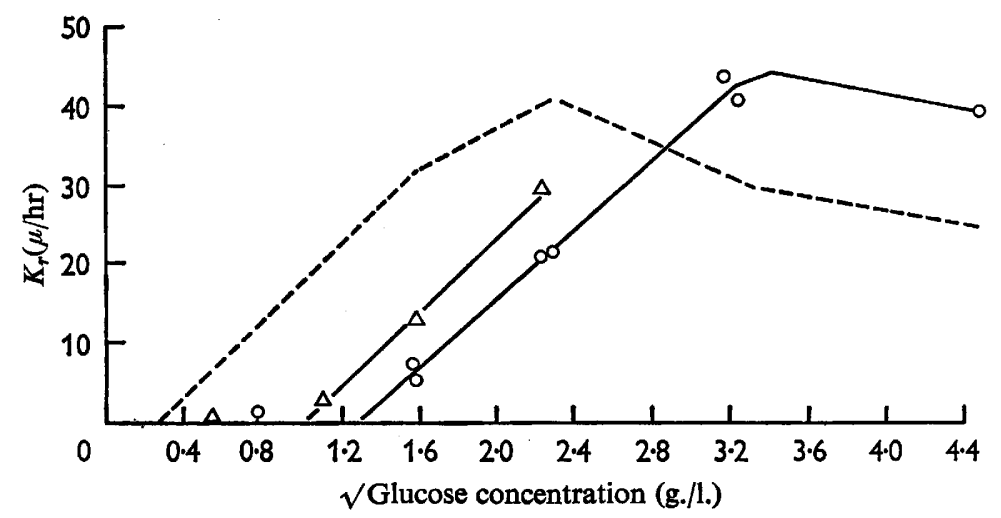

Fig. 7. Effect of increased oxygen and carbon dioxide partial pressures on initial radial growth rates $\left(K_{r}\right)$ of Escherichia coli colonies. $O$, In $100 \%(\mathrm{v} / \mathrm{v})$ oxygen at $1 \mathrm{~atm}$. pressure; $\triangle$, in $95.5 \%(\mathrm{v} / \mathrm{v})$ oxygen $+4.5 \%(\mathrm{v} / \mathrm{v})$ carbon dioxide at $1 \mathrm{~atm}$. pressure. The broken line (from Fig. 4) shows the radial growth rate in air or air $+4.5 \%(\mathrm{v} / \mathrm{v})$ carbon dioxide at $1 \mathrm{~atm}$. pressure. Medium DMA; temperature $37^{\circ}$.

that is, $K_{r}$ was directly proportional to the square root of the glucose concentration, the proportionality factor being the same as in air. However, with oxygen at $1 \mathrm{~atm}$. pressure the initial radial growth rate $\left(K_{r}\right)$ was directly proportional to the square root of the initial glucose concentration $\left(s_{0}\right)$ over a much wider range (1.7-10 g./1.) than in $1 \mathrm{~atm}$. pressure of air $(0 \cdot 1-2.5 \mathrm{~g}$. $/ 1$.$) . This result indicates that in air the depar-$ ture from the linear relation between $K_{r}$ and the square root of the glucose concentration was due to the oxygen diffusion rate becoming growth-limiting.

The absence of $\mathrm{CO}_{2}$ from the oxygen gas phase might have been responsible for the inhibition by oxygen at low glucose concentrations. In a repeat experiment an atmosphere of $95 \%(\mathrm{v} / \mathrm{v}) \mathrm{O}_{2}+4.5 \%$ (v/v) $\mathrm{CO}_{2}$ was used. The results given in Fig. 7 show that the addition of $\mathrm{CO}_{2}$ partially removed the inhibition, but still a glucose concentration of $0.86 \mathrm{~g} . / 1$. was necessary to overcome the inhibition by oxygen. In air an increase in the $\mathrm{CO}_{2}$ partial pressure to $4.5 \%(\mathrm{v} / \mathrm{v})$ did not affect the colony growth rate when it was glucose-limited (Fig. 4).

It is apparent that with increased oxygen partial pressure the lag concentration $\left(s_{i}\right)$ was increased. One would expect that an inhibitory condition, such as over-optimal oxygen partial pressure, which could be overcome by the culture's metabolic activity, would be made manifest in a liquid culture by a lag period before growth. Hence it is believed that the factor $\sqrt{ } s_{i}$ in equation (11) is related to the lag period which would be produced in a liquid culture. 


\section{Effect of decreasing maximum specific growth rate}

To determine the influence of specific growth rate on the colony radial growth rate the specific growth rate was varied by the addition of sulphanilamide as an inhibitor. Sulphanilamide (at low concentrations) in aerobic liquid cultures had the effect of decreasing the maximum specific growth rate $\left(\alpha_{m}\right)$ of Escherichia coli. The maximum specific growth rate was determined by shake-flask culture in the same batch of DMA medium (+ glucose, $5 \mathrm{~g}$./1.) and with the same inoculum culture as were used for the plate cultures. The glucose concentration for the colony growth was $1 \mathrm{~g}$./1. so that the growth was glucose-limited and not oxygen-limited. In one experiment sulphanilamide alone was added to the medium. In another experiment sulphanilamide $+p$-aminobenzoic acid (PABA) was added to vary the specific growth rate. With the PABA

Table 1. Effect of sulphanilamide on specific growth rate and on the initial colony radial growth rate of Escherichia coli, laboratory strain $\mathbf{B}$

Medium DMA glucose $1 \mathrm{~g} . / \mathrm{l}$; incubation temperature $37^{\circ}$.

\begin{tabular}{|c|c|c|c|c|}
\hline $\begin{array}{l}\text { Sulphanilamide } \\
(\mu \mathrm{g} . / \mathrm{ml} .)\end{array}$ & $\begin{array}{l}\text { Maximum speci- } \\
\text { fic growth rate } \\
\left(\alpha_{m} \mathrm{hr}^{-1}\right)\end{array}$ & $\begin{array}{c}\text { Initial colony } \\
\text { radial growth } \\
\text { rate } \\
\left(K_{r}, \mu / \mathrm{hr}\right)\end{array}$ & $K_{r} / \alpha_{m}$ & $K_{r} / \sqrt{ } \alpha_{m}$ \\
\hline \multicolumn{5}{|c|}{$p$-Aminobenzoic acid absent } \\
\hline $\begin{array}{l}0 \\
6 \cdot 25 \\
12 \cdot 5 \\
25 \cdot 0 \\
50 \cdot 0 \\
100 \cdot 0\end{array}$ & $\begin{array}{c}0.615 \\
0.674 \\
0.510 \\
0.351 \\
- \\
-\end{array}$ & $\begin{array}{r}13.4 \\
12.6 \\
11.6 \\
10 \cdot 2 \\
10.4 \\
6.2\end{array}$ & $\begin{array}{c}21 \cdot 8 \\
18 \cdot 7 \\
22 \cdot 7 \\
29 \cdot 1 \\
- \\
-\end{array}$ & $\begin{array}{c}17 \cdot 1 \\
15 \cdot 4 \\
16 \cdot 2 \\
17 \cdot 2 \\
- \\
-\end{array}$ \\
\hline \multicolumn{5}{|c|}{$p$-Aminobenzoic acid $(0.25 \mu \mathrm{g} . / \mathrm{ml}$.$) present$} \\
\hline $\begin{array}{r}0 \\
200 \\
400 \\
800\end{array}$ & $\begin{array}{l}0.661 \\
0.606 \\
0.553 \\
0.517\end{array}$ & $\begin{array}{l}14 \cdot 4 \\
14 \cdot 9 \\
14 \cdot 3 \\
12 \cdot 9\end{array}$ & $\begin{array}{l}21 \cdot 8 \\
24 \cdot 6 \\
25 \cdot 9 \\
24 \cdot 9\end{array}$ & $\begin{array}{l}17 \cdot 7 \\
19 \cdot 2 \\
19 \cdot 2 \\
18 \cdot 0\end{array}$ \\
\hline
\end{tabular}

addition much greater concentrations of sulphanilamide were, of course, required. The results are given in Table 1. With only sulphanilamide added, specific growth rates could not be measured in liquid cultures with sulphanilamide concentrations greater than $25 \mu \mathrm{g}$. $/ \mathrm{ml}$. because of erratic variations in the growth rate. With sulphanilamide + PABA the range of specific growth rates obtained was much smaller than with sulphanilamide alone. It was concluded that within experimental error the ratio of initial colony radial growth rate to the square root of the maximum specific growth rate $\left(K_{r}: \sqrt{ } \alpha_{m}\right)$ was constant. With sulphanilamide alone the mean value of the ratio was 16.3 and the maximum deviation $5.5 \%$. In contrast, the ratio $K_{r}: \alpha_{m}$ showed an upward trend in its value and the maximum deviation from the mean value (23.1) was $20.6 \%$. With sulphanilamide + PABA the mean value of the ratio $K_{r}: \sqrt{ } \alpha_{m}$ was 18.5 and the maximum deviation $4.3 \%$; in contrast, the maximum deviation from the mean value of the ratio $K_{r}: \alpha_{m}$ was $9.7 \%$. From these experiments it was concluded that relation (11) for the initial radial growth rate may be modified to

where $k_{2}$ is a constant.

$$
K_{r}=k_{2}\left(\sqrt{ } s_{0}-\sqrt{ } s_{i}\right) \sqrt{ } \alpha_{m}
$$




\section{Effect of temperature on initial colony radial growth rate}

Changes in the temperature of incubation were made, primarily as an alternative means of varying the growth rate of the organisms. For these experiments an organism was adapted to the growth temperature by growing the inoculum at the temperature required for the growth measurements. A low glucose concentration $(1 \mathrm{~g} . / 1$. was used for the colony growth to ensure that there was excess of oxygen and that growth was glucose-limited. The effects of the temperature changes on the specific growth rates and on the colony radial growth rates for glucose-limited growth are shown in Table 2. Only in the case of Streptococcus faecalis did the colony radial

Table 2. Effect of temperature on initial colony radial growth rate and on the specific growth rate of three bacteria

\begin{tabular}{|c|c|c|c|}
\hline Temp. & $\begin{array}{l}\text { Maximum speci- } \\
\text { fic growth rate } \\
\left(\alpha_{m}, \mathrm{hr}^{-1}\right)\end{array}$ & $\begin{array}{l}\text { Initial colony } \\
\text { radial growth } \\
\text { rate }\left(K_{r}, \mu / \mathrm{hr}\right)\end{array}$ & $K_{r} / \sqrt{ } a_{m}$ \\
\hline \multicolumn{4}{|c|}{ Escherichia coli (glucose, $1 \cdot 1 \mathrm{~g} . / 1$. ) } \\
\hline $\begin{array}{l}37^{\circ} \\
30^{\circ} \\
25^{\circ}\end{array}$ & $\begin{array}{l}0.70 \\
0.445 \\
0.282\end{array}$ & $\begin{array}{l}20 \cdot 0 \\
20 \cdot 4 \\
18 \cdot 0\end{array}$ & $\begin{array}{l}24 \cdot 9 \\
31 \cdot 1 \\
33 \cdot 9\end{array}$ \\
\hline \multicolumn{4}{|c|}{ Klebsiella aerogenes (glucose, 1.0 g./1.) } \\
\hline $\begin{array}{l}37^{\circ} \\
30^{\circ} \\
25^{\circ}\end{array}$ & $\begin{array}{l}0.49 \\
0.72 \\
0.48\end{array}$ & $\begin{array}{l}23 \cdot 3 \\
26 \cdot 2 \\
28 \cdot 3\end{array}$ & $\begin{array}{l}33 \cdot 3 \\
30 \cdot 9 \\
40 \cdot 8\end{array}$ \\
\hline \multicolumn{4}{|c|}{ Streptococcus faecalis (glucose, 1.0 g./1.) } \\
\hline $\begin{array}{l}37^{\circ} \\
31^{\circ} \\
25^{\circ}\end{array}$ & $\begin{array}{l}1.36 \\
1.05 \\
0.65\end{array}$ & $\begin{array}{l}22 \cdot 8 \\
21 \cdot 7 \\
17 \cdot 8\end{array}$ & $\begin{array}{l}19 \cdot 5 \\
20 \cdot 6 \\
22 \cdot 1\end{array}$ \\
\hline
\end{tabular}

growth rate always reflect the change in specific growth rate. The ratio $K_{r}: \sqrt{ } \alpha_{m}$ did not vary by more than $5 \%$ from the mean for $S$. faecalis, but there was an upward trend in the ratio with decrease in temperature of incubation. The upward trend in the ratio $K_{r}: \sqrt{ } \alpha_{m}$ with decrease in temperature was much more marked in the cases of Klebsiella aerogenes and Escherichia coli; in fact, so much so, that a decrease in the specific growth rate was not always reflected in the colony radial growth rate. The upward trend in the ratio $K_{r}: \sqrt{ } \alpha_{m}$ was interpreted to mean that the coefficient $k_{2}$ in (12) was temperature-dependent. The results show that the optimum temperature for colony radial growth rate might be up to $5^{\circ}$ lower than that for the specific growth rate.

In early experiments with Escherichia coli colonies the plates were not dried, and with this condition occasionally a curious temperature effect was noted. At $25^{\circ}$ the initial radial growth rate of the colony might be four times greater than the normal value which was obtained on dried plates. Such anomalous behaviour was not shown by Klebsiella aerogenes under the same condition or by Escherichia coli when the plates were dried or when the temperature was $30^{\circ}-37^{\circ}$. It is suggested that this more rapid spread of the $E$. coli colony associated with a moist plate and a low temperature was an expression of motility. 


\section{DISCUSSION}

From this work on colony growth of three diverse types of bacteria (Gram-negative motile rods, Gram-negative non-motile rods, Gram-positive cocci in chains) on the surface of nutrient agar the following principles are formulated. (1) Large colonies $(2 \mathrm{~mm}$. or more in diameter) increase their radius over a $12 \mathrm{hr}$. period at a nearly constant rate. (2) The initial radial growth rate of large colonies (after about $12 \mathrm{hr}$ development) is directly proportional to the square root of the initial concentration of glucose when this is the growth-limiting nutrient and oxygen is in excess. By analogy one would expect the initial radial growth rate of the colony to be proportional to the square root of the concentration of the growth-limiting nutrient in the agar irrespective of its nature, provided the oxygen relation is constant. (3) When the growth rate is varied by an inhibitor such as sulphanilamide, other things being equal, the initial radial growth rate of the colony is proportional to the square root of the specific growth rate (or inversely proportional to the doubling time).

Changes in maximum specific growth rate brought about by temperature change were not always reflected in colony radial growth rates. This effect, attributed to a change in the coefficient $k_{2}$ of relation (12) cannot be accounted for. It should be noted that the temperature range investigated was relatively narrow (range about $10^{\circ}$ near the optimum growth temperature) and over the full temperature range for growth, the deviation in the responses of colony radial growth rate and of specific growth rate might not be so marked. The measurement of colony radial growth rate might have advantages over liquid cultures for the determination of the temperature range for growth of bacteria.

A gradual decrease in the colony radial growth rate was apparent over longer periods $(24 \mathrm{hr})$. This is taken to be a reflection of a gradual decrease in the rate of nutrient diffusion with time. The recession from the agar colony interface of the level at which the nutrient concentration had the initial value $\left(s_{0}\right)$ means that eventually the agar depth should affect the colony radial growth rate. When the initial glucose concentration was $1 \mathrm{~g} . / 1$. the initial colony radial growth rate became independent of the agar depth when this exceeded $3.5 \mathrm{~mm}$. (the depth given by $20 \mathrm{ml}$. of agar in the $9 \mathrm{~cm}$. Petri dish). To ensure better reproducibility of maximum initial colony growth rate it would be advisable to make the agar depth $5 \mathrm{~mm}$., this would require about $35 \mathrm{ml}$. agar per dish. There appears to be a case for using a Petri dish of $5 \mathrm{~cm}$. diameter in which the optimum depth of agar would be achieved with only $10 \mathrm{ml}$. agar medium.

Two types of growth inhibition were characterized by the colony growth technique. One was the restriction of specific growth rate by sulphanilamide. The other type of inhibition was made manifest in the colony radial growth rate expression (11) by the term $\sqrt{ } s_{i}$. This effect is thought to reflect the type of inhibition which in liquid cultures causes a lag in growth, that is, an inhibitory condition which the organisms can overcome by their own action.

The almost complete inhibition of the growth of Escherichia coli laboratory strain B 3 by a partial pressure of $1 \mathrm{~atm}$. oxygen $(100 \%, \mathrm{v} / \mathrm{v}$ has not been previously noted. Some inhibition of surface colony growth of $E$. coli and of other bacteria by oxygen at $1 \mathrm{~atm}$. pressure was reported by Wiseman, Violago, Roberts \& Penn (1966), but with liquid cultures they obtained conflicting results. These results of Wiseman et al. are difficult to interpret because it is not clear what the growth-limiting nutrient was 
and whether the inhibition was a reflexion of a growth lag or of a decrease in specific growth rate. If the growth-limiting factors in the surface colony are rigorously defined then measurement of colony radial growth rate might be advantageous for the kinetic study of the influence of gases on microbial growth.

Other applications of the quantitative colony growth technique suggest themselves. Maximum specific growth rates $\left(\alpha_{m}\right)$ of different strains of an organism could be compared by means of the expression,

$$
\frac{\left(K_{r}\right)_{A}^{2}}{\left(K_{r}\right)_{B}^{2}}=\frac{\left(\alpha_{m}\right)_{A}}{\left(\alpha_{m}\right)_{B}}
$$

where $K_{r}$ represents initial colony radial growth rate and the subscripts $A$ and $B$ refer to two strains of an organism. This method would be convenient for growth-rate determinations on large numbers of strains as in taxonomical studies. However, for relation (13) to apply, the growth-limiting nutrient should be known to be the same and the same diffusion conditions should apply.

In conventional routine bacteriological plating media the growth-limiting factor is unknown. To avoid an oxygen lack when glucose is the energy source the concentration of glucose should be much lower than is usually the case in routine media for agar plates. For this purpose, in the case of Escherichia coli the concentration of glucose should be less than 2.5 g./1. Again, with routinely used glucose concentrations (e.g. 10-20 g./1.) it appears that growth of colonies is commonly limited by toxic products.

Other applications of the colony growth technique might be based on its ability to estimate growth-limiting substrate $\left(s_{0}\right)$ by relation (12). The method may therefore be useful for the microbiological assay of amino acids, vitamins and other growth factors. The concentration of inhibitors might be determined either by their effect on the initial colony radial growth rate, or on the 'lag concentration' $s_{i}$ in relation (12).

According to the model for colony growth initially proposed the radial growth rate may, from relation (10), be expressed by

$$
K_{r}=w \alpha / 2
$$

For Escherichia coli growing on medium DMA + glucose the maximum value of the specific growth rate $(\alpha)$ is $0.70 \mathrm{hr}^{-1}$. When this value is inserted in (14), then with $K_{r}=32 \mu / \mathrm{hr}$ (glucose concentration $2.5 \mathrm{~g} . / 1$.), $w=91.4 \mu$. Therefore a very small peripheral zone in which the organisms grow at the maximum rate will account for the radial spread of the colony. The model accounts for the constant radial growth rate which is shown by fungal colonies. The gradual decrease with time of the radial growth rate of the bacterial colony is not accounted for. To elucidate the problem further a quantitative analysis of the nutrient diffusion rate would be a great help.

Measurements of the thickness of colonies were not attempted. However, rough visual inspection confirmed that with glucose-limited growth the thickness of the colonies studied here was of microscopic dimensions, as predicted theoretically.

In general, it appears that quantitative growth studies can be extended to microbial colonies in order to obtain knowledge about the reaction of the organism to its environment. The development of a colony is made complex by its possible differentiation into growing and non-growing parts and sometimes into a sporulating part. In the model proposed for the microbial colony, the growing zone may be seen to be analogous to the meristem in a plant tissue, and one could regard the colony of a 
micro-organism as a simple model of a differentiating tissue in which one can control the environmental factors and thus discuss their effects in quantitative terms.

\section{REFERENCES}

Brancato, F. P. \& Golding, N. S. (1953). The diameter of the mould colony as a reliable measure of growth. Mycologia 45, 848.

FAWCETT, H. S. (1925). Maintained growth rates in fungus cultures of long duration. Ann. appl. Biol. $12,191$.

HiLl, A. V. (1928). The diffusion of oxygen and lactic acid through tissues. Proc. R. Soc. B 104, 39. Khatrat, O. (1963). A simple spacer for Petri plates. J. appl. Bact. 26, 232.

MONOD, J. (1942). Recherches sur la croissance bactérienne, 2nd. ed. Paris: Herman.

PIRT, S. J. (1957). The oxygen requirement of growing cultures of an Aerobacter species determined by means of the continuous culture technique. J. gen. Microbiol. 16, 59.

PIRT, S. J. (1965). The maintenance energy of bacteria in growing cultures. Proc. R. Soc. B 163, 224.

PIRT, S. J. (1966). A theory of the mode of growth of fungi in the form of pellets in submerged culture. Proc. R. Soc. B 166, 369.

Plomiey, N. J. B. (1959). Formation of the colony in the fungus Chaetomium. Aust. J. Biol. Sci. 12, 53.

Ryan, F. J., Beadle, G. W. \& TATum, E. L. (1943). The tube method of measuring the growth rate of Neurospora. Am. J. Bot. $30,784$.

Wiseman, G. M., Violago, F. C., Roberts, E. \& Penn, I. (1966). The effect of hyperbaric oxygen upon aerobic bacteria. I. In vitro studies. Can. J. Microbiol. 12, 521. 